\title{
Dynamic Synchronous Phasor Measurement Algorithm Based on Compressed Sensing
}

\author{
Huanan $\mathrm{Yu}^{{ }^{*}}$, Yongxin $\mathrm{Li}^{1}$ and $\mathrm{Yao}_{\mathrm{Du}}{ }^{2}$ \\ ${ }^{1}$ College of Electrical Engineering, Northeast Electric Power University \\ Jilin, 132012, China \\ [e-mail: yhn810117@163.com] \\ ${ }^{2}$ State Grid East Inner Mongolia Electric Power Supply Co., Ltd. Tongliao Power Supply Company Tongliao, \\ 028000, China \\ [e-mail: 1845580185@qq.com] \\ *Corresponding author: Huanan Yu
}

Received September 30, 2018; revised May 12, 2019; revised August 29, 2019; accepted September 11, 2019; published January 31, 2020

\begin{abstract}
The synchronous phasor measurement algorithm is the core content of the phasor measurement unit. This manuscript proposes a dynamic synchronous phasor measurement algorithm based on compressed sensing theory. First, a dynamic signal model based on the Taylor series was established. The dynamic power signal was preprocessed using a least mean square error adaptive filter to eliminate interference from noise and harmonic components. A Chirplet overcomplete dictionary was then designed to realize a sparse representation. A reduction of the signal dimension was next achieved using a Gaussian observation matrix. Finally, the improved orthogonal matching pursuit algorithm was used to realize the sparse decomposition of the signal to be detected, the amplitude and phase of the original power signal were estimated according to the best matching atomic parameters, and the total vector error index was used for an error evaluation. Chroma 61511 was used for the output of various signals, the simulation results of which show that the proposed algorithm cannot only effectively filter out interference signals, it also achieves a better dynamic response performance and stability compared with a traditional DFT algorithm and the improved DFT synchronous phasor measurement algorithm, and the phasor measurement accuracy of the signal is greatly improved. In practical applications, the hardware costs of the system can be further reduced.
\end{abstract}

Keywords: Synchronous phasor measurement, dynamic conditional compressive sensing, Taylor series, sparse decomposition 


\section{Introduction}

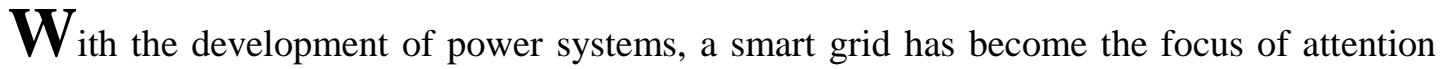
globally. A smart grid (SG) is a data communications network which is integrated with the power grid to collect and analyze data that are acquired from transmission lines, distribution substations, and consumers[1]. SG is based on an integrated and high-speed two-way communication network, applying advanced sensing and measurement technology, equipment technology, control methods, and decision support systems to realize a reliable, safe, economical, efficient, friendly, and safe grid[2,3]. The core of a smart grid mainly includes the following aspects: a real-time collection and transmission of the power system data using sensors [4], a data integration system, and a data collection system [5, 6]. In addition, it has the ability to conduct a self-analysis, that is, based on data developed to carry out a relevant analysis for optimizing the operation and management of the grid. The configuration of a number of sensors in a smart grid directly affects the cost input [7]. We hope we can achieve a balance between cost, data communications delay, and energy[8].

As an important platform for a real-time estimation and the dynamic monitoring of the smart grid status, wide area measurement systems (WAMS) have been widely used in power system security and stability control, system parameter identification, and fault analysis [9]. The core of WAMS is the phasor measurement unit (PMU) based on global positioning technology, which can realize the synchronous transmission, measurement, and analysis of phasors in the power system. The PMU is the most important component for obtaining dynamic real-time monitoring data and an accurate system estimation [10, 11]. To a large extent, the accuracy of a PMU device depends mainly on the algorithm applied. It is therefore important to improve the accuracy and reliability of the synchronous phasor measurement algorithm.

At present, many synchronous phasor measurement algorithms have been widely used, such as the discrete Fourier transform (DFT) method [12, 13], zero-crossing detection method [14, 15], and a digital filter method [16, 17]. Because the DFT algorithm is suitable for fast computations and has good harmonic suppression characteristics, it has an increased value of application under static conditions, hence its wide use in synchronous phasor measurements. However, DFT-like algorithms also have certain drawbacks. For example, when the system operates under asynchronous sampling, the existing DFT algorithm tends to produce errors in the amplitude and phase measurements, and such errors increase sharply with the increase in asynchronization, resulting in frequency spectrum leakage and a fence effect. These results fail to meet the requirements of the actual application [18]. Moreover, the existing DFT algorithm is often affected by the dynamic conditions of the system, such as low-frequency oscillations, resulting in large errors in the phasor measurements, increasing the difficulty to meet the needs of practical applications [19].

In manuscript [20] based on the improved DFT algorithm, a phase angle error equation has been deduced. Using the phase angle difference to track the measurements of the signal frequency, the DFT results from tracking the frequency are divided into an integer part and score, and through the equivalent replacement of the fractional part a correction is carried out to reduce the errors from the frequency spectrum leakage and fence effect.

In addition, the authors of [21] designed a complex bandpass filtering algorithm based on a Gaussian window decoupling modulation function, which has good stability and response speed. In [22], using the Taylor series and a strong tracking filter, a strong tracking Taylor-Kalman filter (STKF) dynamic phasor measurement method with good measurement accuracy is proposed. 
The above methods have their own characteristics, but based on the fundamental principle, the common point is that the Nyquist sampling theorem is used to synchronously collect the voltage and current signals of different nodes and busbars in the system. For a high sampling frequency, this is bound to bring about huge amounts of data, putting heavy pressure on the real-time transmission and processing of the signals.

To adapt to the dynamic conditions of a power system, this manuscript proposes a new algorithm for optimizing the synchronous phasor measurements based on the Taylor series and compressive sensing technology. The core idea of this method is to establish a dynamic signal model based on the Taylor series, and effectively filter out the interference components through preprocessing. A phasor optimization measurement of the power signal is then realized using compressed sensing. Compared with the traditional DFT algorithm and the improved DFT algorithm in [20], the compressed sensing used in this manuscript can significantly reduce the amount of data collection, improve the operation efficiency of the system, and avoid a spectrum leakage and fence effect. The method not only effectively improves the accuracy of the phasor measurement, it also improves the anti-interference and dynamic performance of the algorithm, reduces the number of iterations in the signal processing, and lays a good foundation for the application of synchronous phasor measurement devices in WAMS.

\section{Power Signal Dynamic Model}

According to the IEEE C37.118-2005 standard [23], the standard form of a power signal is

$$
x(t)=A \cos (2 \pi f t+\phi)
$$

where $A$ is the amplitude, $f$ is the fundamental frequency, and $\phi$ is the initial phase angle.

It can be seen from equation (1) that this form can only characterize a static signal model whose amplitude and phase parameters do not change. In practice, because a power system often undergoes dynamic changes, the amplitude and phase of the signal will change. The traditional static signal model cannot accurately represent the time-variance of the fundamental signal component. Therefore, this manuscript attempts to use the complex signal $X(t)$ to characterize the dynamic phasor of the power signal. Thus, equation (1) can be expressed as a complex exponential function.

$$
x(t)=\frac{1}{2}\left[X(t) e^{j 2 \pi f t}+X^{*}(t) e^{-j 2 \pi f t}\right]
$$

where

$$
X(t)=\sqrt{2} A(t) e^{j \phi(t)}
$$

In equation (3), * is the conjugation, $A(t)$ and $\phi(t)$ are the amplitude and phase of a dynamic signal, respectively, at moment $t$.

For the sake of analysis, this manuscript used the Taylor series to approximate the power magnitude $A(t)$ and phase $\phi(t)$ of a signal at moment $t$, as follows. 
which is similar to

$$
\begin{aligned}
A(t) & =\sum_{n=0}^{+\infty} \frac{1}{n !} A^{(n)} t^{n} \\
& =A_{0}+A^{\prime} t+\frac{1}{2 !} A^{\prime \prime} t^{2}+\cdots+\frac{1}{n !} A^{(n)} t^{n}
\end{aligned}
$$

$$
\begin{aligned}
\phi(t) & =\sum_{n=0}^{+\infty} \frac{1}{n !} \phi^{(n)} t^{n} \\
& =\phi_{0}+\phi^{\prime} t+\frac{1}{2 !} \phi^{\prime \prime} t^{2}+\cdots+\frac{1}{n !} \phi^{(n)} t^{n}
\end{aligned}
$$

Here, $A_{0}$ is the power signal amplitude at a reference time $t_{0} \cdot t_{0}$ is usually equal to the center time of the sampled data window, $A^{(n)}$ is $A(t)$ at the $n$ order derivative of time $t_{0}, \phi_{0}$ is the power signal phase of reference time $t_{0}$, and $\phi^{(n)}$ is $\phi(t)$ at the $n$ order derivative of time $t_{0}$.

According to the approximate characteristics of the Taylor series, the larger the value of $n$ is, the closer the polynomial is to the target function, i.e., the larger of $n$ is, the closer the values of $A(t)$ and $\phi(t)$ are to the true phasor value at moment $t$. However, when the value of $n$ increases, the number of computations increases as well, which delays the phasor measurement algorithm. Under normal circumstances, it can be considered that the parameter change of the power signal under dynamic conditions is slow, and the low-order derivative can represent the dynamic characteristics of the signal. Therefore, in this manuscript, let $n=1$; Thus, the first-order Taylor series is used to approximate the phase value, i.e.,

$$
\begin{aligned}
& A(t)=A_{0}+A^{\prime} t \\
& \phi(t)=\phi_{0}+\phi^{\prime} t
\end{aligned}
$$

with $A^{\prime}$ and $\phi^{\prime}$ representing the magnitude and phase of the power signal of the first derivative at reference moment $t_{0}$.

Thus, an approximate model of the dynamic signal of the power system can be obtained.

$$
\begin{aligned}
x(t)= & \frac{\sqrt{2}}{2}\left[X(t) e^{j 2 \pi f t}+\left(X(t) e^{-j 2 \pi f t}\right)^{*}\right] \\
= & \frac{\sqrt{2}}{2}\left(A_{0}+A^{\prime} t\right) e^{j\left(\phi_{0}+\phi^{\prime} t\right)} e^{j 2 \pi f t}+ \\
& \frac{\sqrt{2}}{2}\left[\left(A_{0}+A^{\prime} t\right) e^{j\left(\phi_{0}+\phi^{\prime} t\right)}\right]^{*} e^{-j 2 \pi f t}
\end{aligned}
$$




\section{Dynamic Synchronous Phasor Measurement Algorithm Based on Compressed Sensing}

To improve the measurement accuracy of the algorithm and save system space and cost, this paper proposes a dynamic synchronous phasor measurement algorithm based on compressive sensing. The overall process of the algorithm is described below.

\subsection{Pretreatment}

In a power grid, noise is one of the important factors affecting the measurement of the power system signal for a synchronous phasor measurement. Particularly when the system is under dynamic conditions, a large amount of noise contained in the signal will seriously affect the accurate measurement of the parameters, such as the amplitude and phase. In addition, when the system fails, it often causes a large amount of harmonic components to be generated in the voltage and current signals, which will also drastically reduce the measurement accuracy of the phasor. Therefore, in this manuscript an adaptive filter is applied based on the least mean square (LMS) criterion [24] to preprocess the power signal, thus filtering out interference signals such as noise and harmonics.

LMS adaptive filters are widely used in the field of power systems owing to their simplicity, robustness, and ease of implementation. In the process of filtering a signal, the signal model of the power system is set as follows:

$$
x(n)=s(n)+v(n)
$$

Here, $x(n)$ represents complex power signals containing harmonics or noise, $s(n)$ represents the ideal power signal, and $v(n)$ represents Gaussian white noise or the harmonic components, which are independent of the ideal power signal. In an adaptive filter based on the LMS criterion, the main idea is to minimize the mean square error $e(n)$ between the filter's output signal $y(n)$ and the expected output signal $d(n)$. Therefore, complex power signals containing components such as harmonics or noise are input into the filtering system and estimated, the process of which is mainly divided into the following steps:

1) Filtering process.

$$
y(n)=w(n)^{T} x(n)
$$

In the formula, $y(n)$ represents the output signal at time $n$, and $w(n)$ represents the filter weight factor.

2) Error calculation process.

In the filtering process, assuming that the expected output signal $d(n)$ is known, the error between the expected signal and the output signal can then be calculated.

$$
e(n)=d(n)-y(n)
$$


The mean square error of which is:

$$
\begin{aligned}
J= & E\left[e(n)^{2}\right]=E\left[d(n)^{2}\right]- \\
& 2 E\left[d(n) w(n)^{T} x(n)\right]+E\left[w(n)^{T} x(n)^{T} w(n)\right]
\end{aligned}
$$

Next, the following is solved:

$$
\begin{aligned}
\hat{\nabla J} & =\frac{\partial\left(e(n)^{2}\right)}{\partial w(n)} \\
& =\left[2 e(n) \frac{\partial e(n)}{\partial w_{1}(n)}, \cdots, 2 e(n) \frac{\partial e(n)}{\partial w_{M}(n)}\right]^{T} \\
& =-2 e(n) x(n)
\end{aligned}
$$

Here, $M$ represents the number of iterations of the LMS algorithm, and $\hat{\nabla} J$ represents the gradient of $M$ iterations.

3) Update the filter coefficient

$$
\begin{aligned}
w(n+1) & =w(n)+2 \mu(-\nabla J) \\
& =w(n)+2 \mu e(n) x(n)
\end{aligned}
$$

In (13), $\mu$ represents the step length factor, and

$$
\nabla J \approx \hat{\nabla} J
$$

Therefore, through equations (9), (10), and (13) above, the effective power signal output by the filter system can be obtained, and the effective power signal is determined to be close to the ideal signal.

\subsection{Algorithm implementation}

The dynamic synchronous phasor measurement algorithm based on compressive sensing theory designed in this manuscript mainly uses an overcomplete dictionary to realize the sparse decomposition of the original power signal, and it matches the tracking using an iterative algorithm, thus achieving an accurate measurement of the phasor value.

\subsubsection{Principle of the algorithm}

In a large power system, although the voltage and current signals have complex and changeable characteristics, the main purpose of synchronous phasor measurements of the power signals is to obtain the amplitude and phase information of the fundamental wave components. Gabor atoms are commonly used to decompose a sparse signal, the expression of which is as follows: 


$$
g_{\gamma}(t)=\frac{1}{\sqrt{s}} g\left(\frac{t-u}{s}\right) \cos (\varepsilon t+\phi)
$$

Here, $g_{\gamma}(t)$ represents Gabor atoms defined by the cosine function parameter set $\gamma$; $g(t)$ represents the Gaussian window function; $s$ is the scaling factor; $u$ is the displacement factor, which is the center of time; $\varepsilon$ is the modulation factor, i.e., the frequency center; $\phi$ represents the phase parameter; And $\gamma=(s, u, \varepsilon)$ is the set of atomic parameters.

The frequency of Gabor atoms does not change with time, but the frequency of power system signals often fluctuates near the power frequency, if the power system signal is sparse by the Gabor atoms, many combinations of Gabor atoms are needed to approximate the dynamic spectrum characteristics of the signal. Thus, in this manuscript, a Chirplet sparse dictionary with a slope factor was constructed to sparse the signal. Compared with the traditional overcomplete Gabor dictionary, the waveform library composed of four-parameter Chirplet atoms can more effectively represent the time-varying signal [25] and the number of iterations of signal processing for various power systems is significantly reduced.

The design process is as follows:

$$
g_{\gamma}=\sqrt{\frac{1}{s_{i}}} g\left(\frac{t-u_{l}}{s_{i}}\right) \exp \left(j\left(\xi_{p}\left(t-u_{l}\right)+\frac{1}{2} c_{q}\left(t-u_{l}\right)^{2}\right)\right)
$$

In the formula, $g_{\gamma}(t)$ represents the Chirplet atoms defined by parameter set $\gamma ; g(t)$ represents the Gaussian window function; $s_{i}$ is the scaling factor; $i=1,2, \cdots, I ; u_{l}$ is the displacement factor; $l=1,2, \cdots, L ; \quad \xi_{p}$ is the frequency shift factor; $p=1,2, \cdots, P ; \quad c_{q}$ represents the linear frequency modulation factor; $q=1,2, \cdots, Q ; \gamma=\left(s_{i}, u_{l}, \xi_{p}, c_{q}\right)$ is the set of atomic parameters; $t$ is the time parameter; and $t=0,1, \cdots, N-1$. According to the application conditions of the algorithm, the search range of $s_{i}, u_{l}, \xi_{p}$, and $c_{q}$ is provided, and the value is evenly selected according to a certain search precision.

The set of atomic parameters $\gamma=\left(s_{i}, u_{l}, \xi_{p}, c_{q}\right)$ can form atoms with different characteristics. For example, when $i=l=p=q=1$, there is $\gamma=\left(s_{1}, u_{1}, \xi_{1}, c_{1}\right)$. At this point, an atom $g_{\gamma_{0}}$ in the overcomplete atomic library is formed:

$$
g_{\gamma_{0}}=\sqrt{\frac{1}{s_{1}}} g\left(\frac{t-u_{1}}{s_{1}}\right) \exp \left(j\left(\xi_{1}\left(t-u_{1}\right)+\frac{1}{2} c_{1}\left(t-u_{1}\right)^{2}\right)\right)
$$

By analogy, we can build a library of atoms with different characteristics. In addition, when building a complete atomic library, it is also necessary to pay attention to the normalization of the atom, i.e., $\left\|g_{\gamma}\right\|=1$, with $\|\cdot\|$ representing the norm of the signal, which is defined as the distance of the signal (a point in the space) to the origin of the spatial coordinate (corresponding to the 0 signal) in the $N$ dimensional Hilbert space $R^{N}$. 
Therefore, for an $N \times 1$ dimensional power signal $x$, we have

$$
D=\left\{g_{\gamma_{0}}, g_{\gamma_{1}}, \cdots, g_{\gamma_{n}}\right\}=\left[\begin{array}{cc}
D_{11} D_{12} \cdots D_{1 n} \\
D_{21} D_{22} \cdots D_{2 n} \\
\vdots & \ddots \\
D_{N 1} D_{N 2} \cdots D_{N n}
\end{array}\right]_{N \times n}
$$

In the formula, $D_{N n}$ is the $N$-th element of the $n$-th atom, $g_{\gamma_{n}}$ is the $n$-th atom in the atomic library, the number of atoms in the entire atomic library is $n=I L P Q$, and $n>>N$, which satisfies the overcomplete feature of the dictionary.

Through the Chirplet overcomplete atomic library $D$ in the above process, the original power signal $x$ becomes sparse. This process can be expressed as

$$
x=D a
$$

where $a$ is a sparse vector.

To reduce the transmission and storage burden of the system, this paper proposes a Gaussian random matrix $\Phi$ to construct the observation matrix and obtain a small amount of measurement data $y$. Let the observation matrix be $\Phi \in R^{M \times N}(M<<N)$, the measurement vector is $y=\Phi x$, namely,

$$
\left[\begin{array}{c}
y_{1} \\
y_{2} \\
\vdots \\
y_{M}
\end{array}\right]_{M \times 1}=\left[\begin{array}{cccc}
\varphi_{11} & \varphi_{12} & \cdots & \varphi_{1 N} \\
\varphi_{21} & \varphi_{22} & \cdots & \varphi_{2 N} \\
\vdots & \ddots & \\
\varphi_{M 1} & \varphi_{M 2} & \cdots & \varphi_{M N}
\end{array}\right]_{M \times N} \cdot\left[\begin{array}{c}
x_{1} \\
x_{2} \\
\vdots \\
x_{N}
\end{array}\right]_{N \times 1}
$$

This process realizes the dimensionality reduction projection of the original power signal $x$ from $N$ to $M$ dimensions, thus achieving the purpose of the data compression.

The sparse decomposition algorithm is the core component of the entire algorithm. In this manuscript, the orthogonal matching pursuit (OMP) algorithm [26] was used to achieve the purpose of decomposing the measured signal. The basic idea is to select the atoms $g_{\gamma}$ that best match the signal $y$ to be decomposed from the overcomplete atomic library, thereby achieving the parameter estimation of the fundamental component, the specific process of which is as follows:

Step 1: Set the decomposition parameters according to the dynamic condition of signal $y$ to be decomposed, given the search range of each decomposition parameter of $s_{i}, u_{l}, \xi_{p}$, and $c_{q}$.

Step 2: Build the atomic library according to equations (16) and (18), and construct an over-complete atomic library $D=\left\{g_{\gamma} \mid \gamma \in\left(s_{i}, u_{l}, \xi_{p}, c_{q}\right)\right\}$ with different characteristics.

Step 3: Conduct the best atomic search. Search for the atom with the largest inner product with the signal $y$, which is the best atom $g_{\gamma_{k}}$, where $k$ is the number of decomposition, $y_{0}$ is the original signal $y$, and when $k>1, y_{k}$ is the residual error signal. In the process of obtaining the best matching atom, owing to the characteristics of the 
sparse signal decomposition, the signal to be decomposed will reach the maximum match on a certain component when conducting an OMP decomposition, the optimal atomic search threshold condition is as follows:

$$
\left|\left\langle y_{k}, g_{\gamma_{k}}\right\rangle\right|=\sup _{\gamma \in \Gamma}\left|\left\langle y_{k}, g_{\gamma_{v}}\right\rangle\right|
$$

where $\left\langle y_{k}, g_{\gamma_{k}}\right\rangle$ is the inner product of the decomposed signal $y$ and its best match atom $g_{\gamma_{k}}, \quad v=0,1, \cdots k$.

Step 4: Decompose the signal. The optimal atom obtained by the search conducted in step 3 sparsely decomposes signal or residual signal, the signal can be expressed as:

$$
y_{k}=\left\langle y_{k}, g_{\gamma_{k}}\right\rangle g_{\gamma_{k}}+y_{k+1}
$$

Where $y_{k+1}$ is the new residual signal.

Step 5: Calculate the residual signal. The residual signals after a sparse decomposition can be obtained using the difference between the original signal and the inner product of successive iterations:

$$
y_{k+1}=y-\sum_{v=0}^{k}\left\langle y_{k}, g_{\gamma_{k+1}}\right\rangle g_{\gamma_{k+1}}
$$

Step 6: Judge the threshold. Considering the time complexity and practicability of the algorithm, sparse decomposition sets two termination thresholds of the maximum decomposition number termination and minimum residual signal termination. If the decomposition times reach the preset upper threshold or the residual signals reach the preset lower threshold, the decomposition is terminated; otherwise, return to step 3 . Therefore, the final signal of the complete decomposition process can be expressed as follows:

$$
y=\sum_{k=0}^{n}\left\langle y, g_{\gamma_{k}}\right\rangle g_{\gamma_{k}}+y_{n+1}
$$

After the signal $y$ is obtained, the original signal $x$ can be obtained through the signal's matching pursuit. In the process of finding the best matching atom, to make the atomic library contain as many atomic numbers and types as possible, the process computations are extensive. Therefore, this manuscript uses the fast Fourier transformation (FFT) to improve the OMP algorithm and improve the overall speed of the algorithm.

First, based on the characteristics of the overcomplete atomic library, for the atomic parameters $s_{i}, u_{l}, \xi_{p}$ and $c_{q}$, when $i$ in $s_{i}$ is from $i=1$ to $i=\mathrm{I}$, the inner product of the atom and the signal to be decomposed $\left\langle y, g_{\gamma}\right\rangle$ are calculated I times. This process demands extensive computations. Therefore, to improve the overall speed of the algorithm, the I times of inner product operation is converted into a one-time cross-correlation operation between $y$ and $g_{r}$, which can significantly reduce the number of computations, i.e.,

$$
R_{y, g_{r}}=\left\langle y, g_{\gamma_{i}}\right\rangle
$$


Although the conversion of a large number of inner product calculations into a small number of cross-correlation operations improves the overall efficiency of the algorithm, to further reduce the number of computations and algorithm runtime, as well as speed up the process of the cross-correlation operation, this paper proposes the use of an FFT.

Because the majority of the computations of the above algorithm are generated during the calculation of the inner product $\left\langle y, g_{\gamma}\right\rangle$, the FFT is applied to speed up the algorithm process. This can not only quickly achieve cross-correlation operations, it can also significantly improve the speed of the atomic sparse decomposition and thus the entire algorithm process.

\subsubsection{Algorithm evaluation criteria}

In a power system, because the phasor is divided into the amplitude and phase, there is also a difference between the amplitude and phase errors when evaluating the PMU. In the IEEE Std C37.118-2011 standard [27], an effective method for evaluating the phasor measurement error of the synchronous phasor measuring device generally adopts a total vector error (TVE). Using the TVE indicator to measure the PMU is not only more scientific and comprehensive, it also accurately reflects the performance of the algorithm, which is conducive to visually compare the performance of other phasor measurement methods.

The TVE is defined as follows:

$$
T V E=\sqrt{\frac{\left[X_{R}(n)-X_{R}\right]^{2}+\left[X_{I}(n)-X_{I}\right]^{2}}{X_{R}^{2}+X_{I}^{2}}}
$$

where $X_{R}$ is the real part of the ideal signal phasor, $X_{I}$ is the imaginary part of the ideal signal phasor, $X_{R}(n)$ is the actual part of the measured phasor, and $X_{I}(n)$ is the imaginary part of the measured phasor.

The smaller the value of the TVE, the closer the phasor measurement is to the true value, and the more accurate the performance of the phasor measurement algorithm. Therefore, the performance of the algorithm can be accurately measured based on the TVE value.

\section{Simulation Results and Analysis}

In power systems, power signals are often affected by a system failure or instability of the power unit, resulting in a large amount of harmonic components in the signal, or low-frequency oscillations and amplitude or phase steps. Therefore, to accurately evaluate the performance of the algorithm, a representative power signal model was established to simulate the phasor measurement of the actual power system signal under dynamic conditions. In this manuscript, four dynamic signal models were established as experiment objects, namely, low-frequency oscillating signals, harmonic-containing power signals, amplitude step signals, and phase step signals. The signals in this article were generated using a programmable power supply (Chroma PROGRAMMABLE AC SOURCE 61511, as shown in Fig. 1) and analyzed in LABVIEW and MATLAB. 


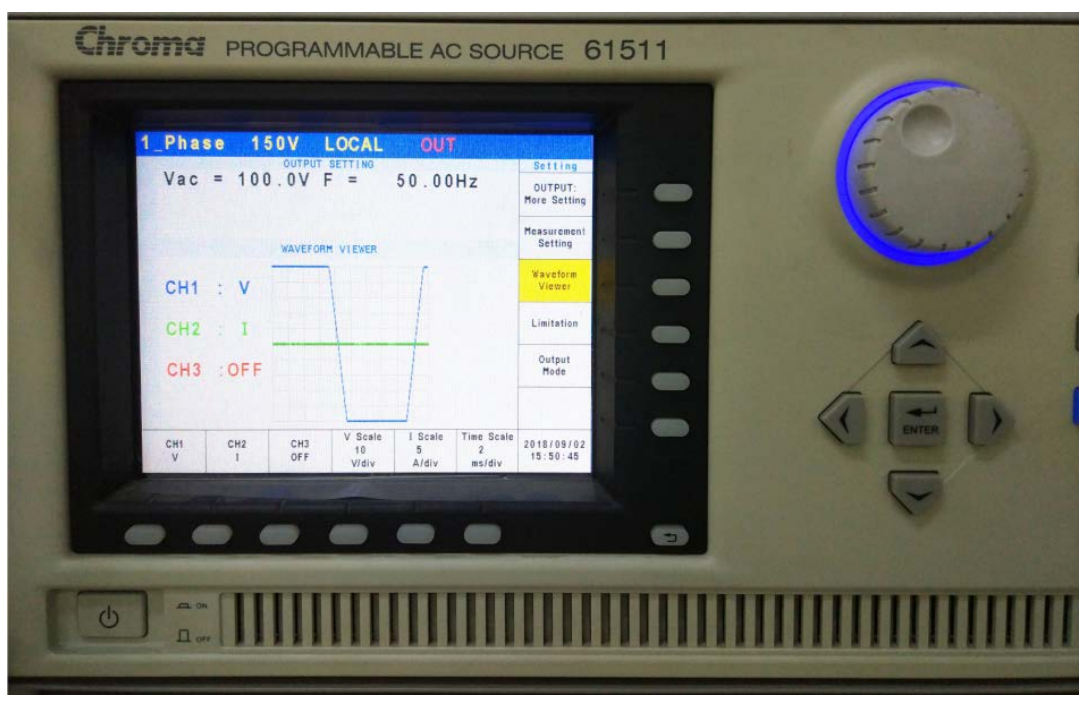

Fig. 1. Chroma 61511

\subsection{Comparison of dictionary parameters}

Using the Chirplet dictionary in the framework of the compressed sensing algorithm and comparing it with a Gabor dictionary, a better Chirplet dictionary is determined as a sparse dictionary, and the corresponding reasons are provided. Two dictionaries were used to compare the sparse reconstruction of power signals containing harmonics generated using Chroma 61511, as shown in Fig. 2.

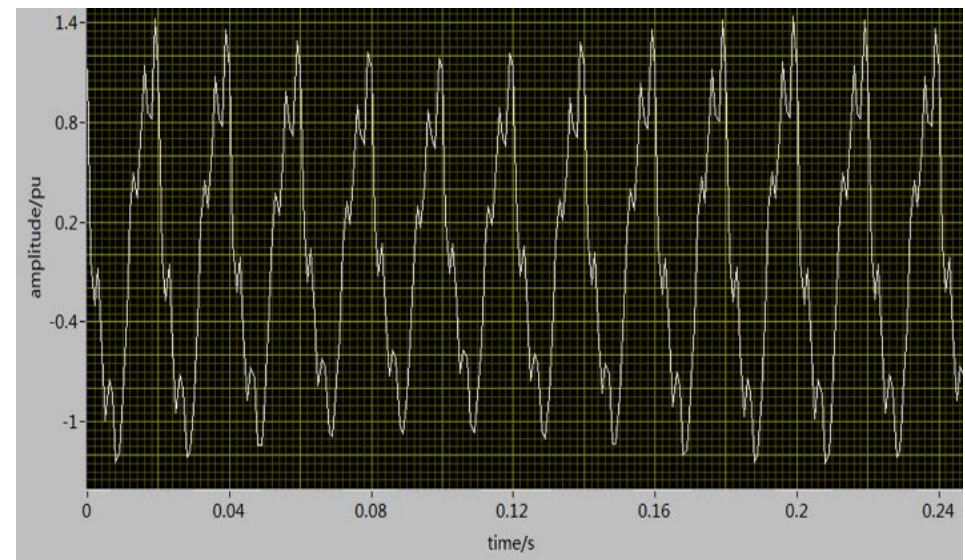

Fig. 2. Harmonic signal

The calculation time and the number of atoms required for sparsity was taken as the index of the sparsity effect, and through a comparison, it can be seen that under the sparse processing of the same signal, the Chirplet dictionary significantly reduces the number of atoms required for the sparse processing, reduces the number of operations, reduces the operation time, and significantly improves the efficiency of the sparsing and reconstruction. The running time comparison for the sparse processing between Gabor and Chirplet dictionary shows in Fig. 3. And the number of atoms required for the sparse processing between Gabor and Chirplet dictionary shows in Fig. 4. 


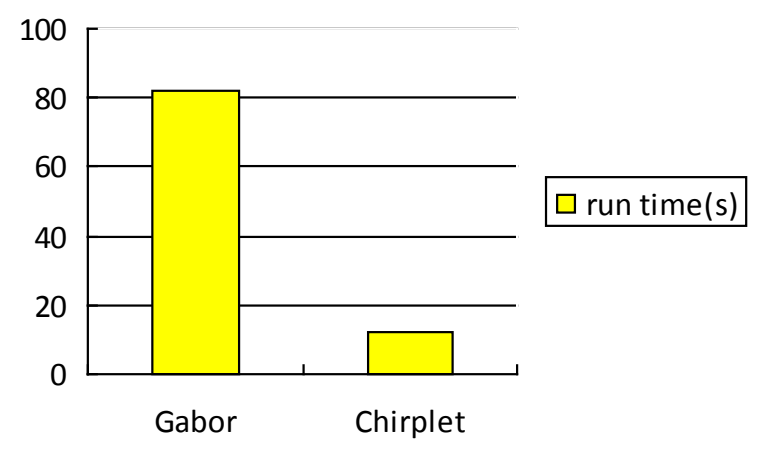

Fig. 3. Running time comparison

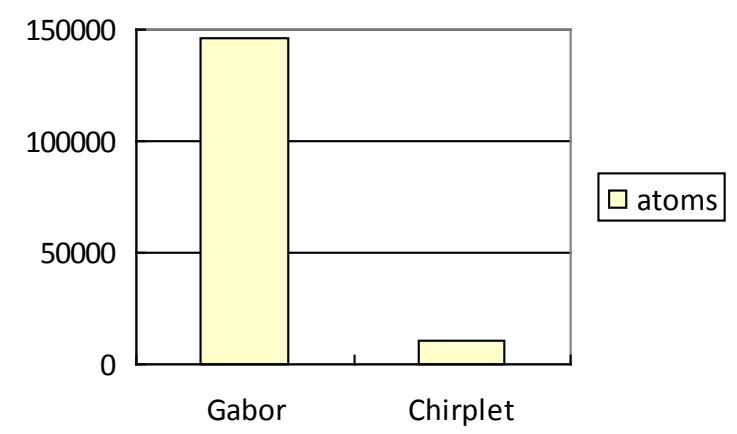

Fig. 4. Comparison of required atomic numbers

\subsection{Phasor measurement of low-frequency oscillation signals}

Under low-frequency oscillation conditions, the signal model was set to

$$
x(t)=a(t) \cdot \cos \left[2 \pi f_{0} t+\phi(t)\right]
$$

where $a(t)$ is the amplitude, $\phi(t)$ is the phase, $f_{0}$ is the fundamental frequency, and $f_{0}=50 \mathrm{~Hz}$.

Because the amplitude and phase of the power signal are usually time-varying, the amplitude and phase were set as $a(t)$ and $\phi(t)$, respectively.

$$
a(t)=a_{0}+a_{1} \cos \left(2 \pi f_{a} t\right)
$$

where $a_{0}=1, a_{1}=0.1, \phi_{0}=\pi / 4, \phi_{1}=0.1$, and $f_{a}=f_{\phi}=50 \mathrm{~Hz}$. The waveform of the oscillating signal is shown in Fig. 5. 


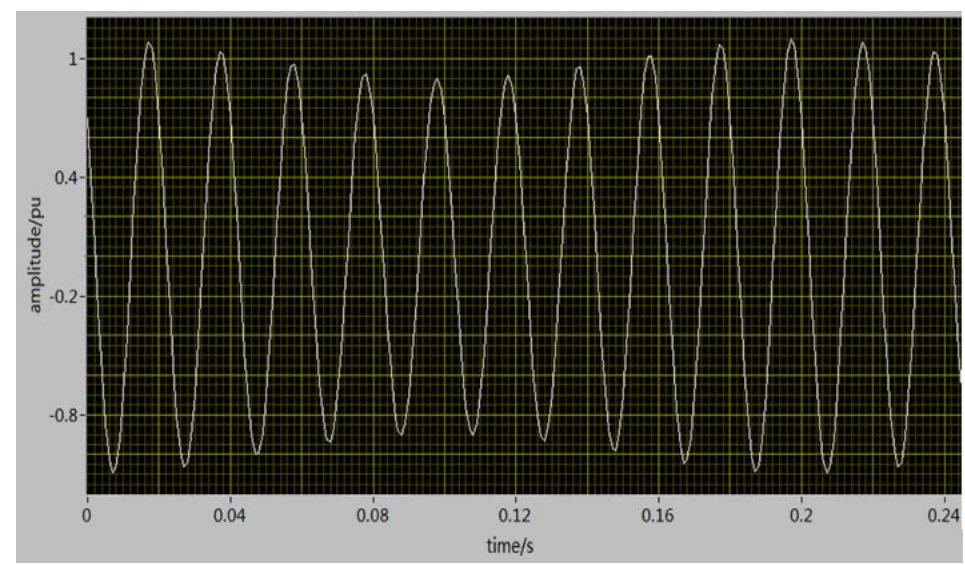

Fig. 5. Low-frequency oscillation signal waveform

In this manuscript, phasor measurements of low-frequency oscillation signals were carried out using the CS algorithm, the traditional DFT algorithm, and the improved DFT algorithm described in [20], and the measured value of the phasor was compared with the theoretical value to obtain the amplitude, phase error, and TVE value at each moment. As can be seen from Fig. 6, the traditional DFT algorithm shows an obvious oscillation phenomenon during the entire phasor measurement process, and both the amplitude and phase errors are large, the maximum value of TVE is approximately 5\%, whereas the error of the amplitude or phase of the improved DFT algorithm is clearly reduced, and the maximum value of the TVE is only approximately $2 \%$. Compared with the improved DFT algorithm, the amplitude and phase errors of the CS algorithm are obviously smaller and more stable, and the TVE value is kept below $0.5 \%$, which meets the precision requirements of the standard IEEE-37.118.1-2011 for the phasor measurement of the oscillation signal of the PMU.

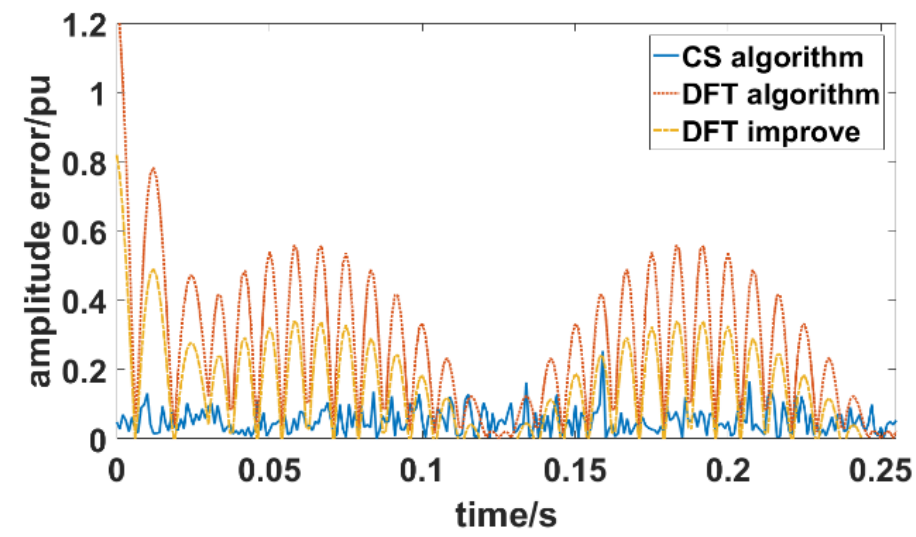

(a) Amplitude measurement error of low-frequency oscillation signal 


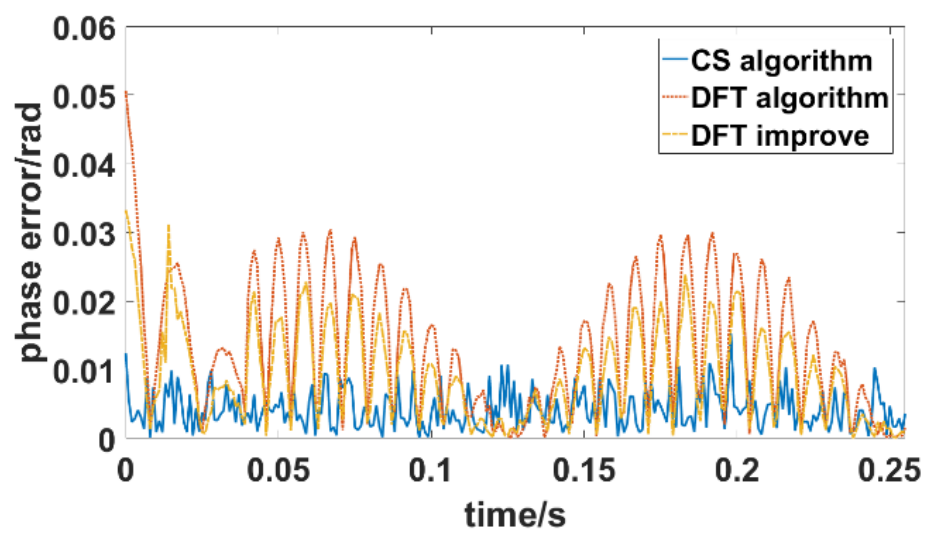

(b) Phase measurement error of low-frequency oscillating signal

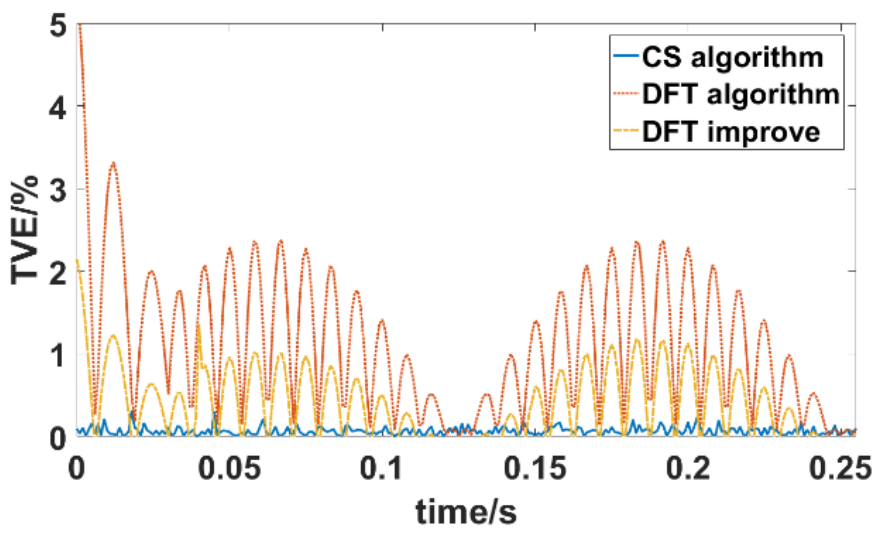

(c) TVE measurement of low-frequency oscillation signals

Fig. 6. CS and DFT algorithm measurement results for low-frequency oscillation signals

\subsection{Phasor measurement of harmonic signals}

Non-linear loads in power systems often cause a distortion of the waveforms of the voltage and current signals, which cause a large amount of harmonic components in the power signal, and are usually also interfered with by noise. It was therefore assumed that the amplitude and phase of the time-varying power signals were included in the noise, along with the third, fifth, and sixth harmonic components. The test signal model is then

$$
\begin{aligned}
x(t)= & a(t) \cos \left[2 \pi f_{0} t+\phi(t)\right]+0.1 a(t) \cos \left[2 \pi 3 f_{0} t+\phi(t)\right]+ \\
& 0.1 a(t) \cos \left[2 \pi 5 f_{0} t+\phi(t)\right]+0.1 a(t) \cos \left[2 \pi 6 f_{0} t+\phi(t)\right] \\
& +R_{n}
\end{aligned}
$$

In the formula below, $R_{n}$ is a Gaussian white noise component with a signal-to-noise ratio of $50 \mathrm{~dB}$.

Because the test signal contains harmonic and interference noise components, such components need to be filtered out for an accurate measurement of the phasor value. A waveform comparison analysis diagram of the oscillating power signal containing harmonics and noise, the original power signal, and the output signal of the filtering system is shown in 
Fig. 7. It can be seen from Fig. 7 that when the original power signal contains harmonics, the LMS adaptive filter can effectively filter out harmonic components and interference noise, and obtain a more precise output signal.

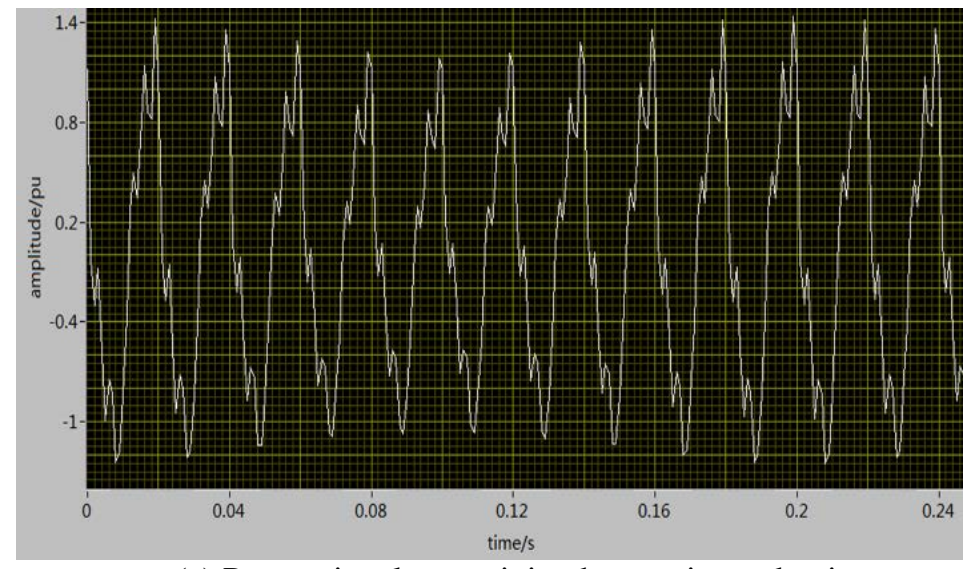

(a) Power signals containing harmonics and noise

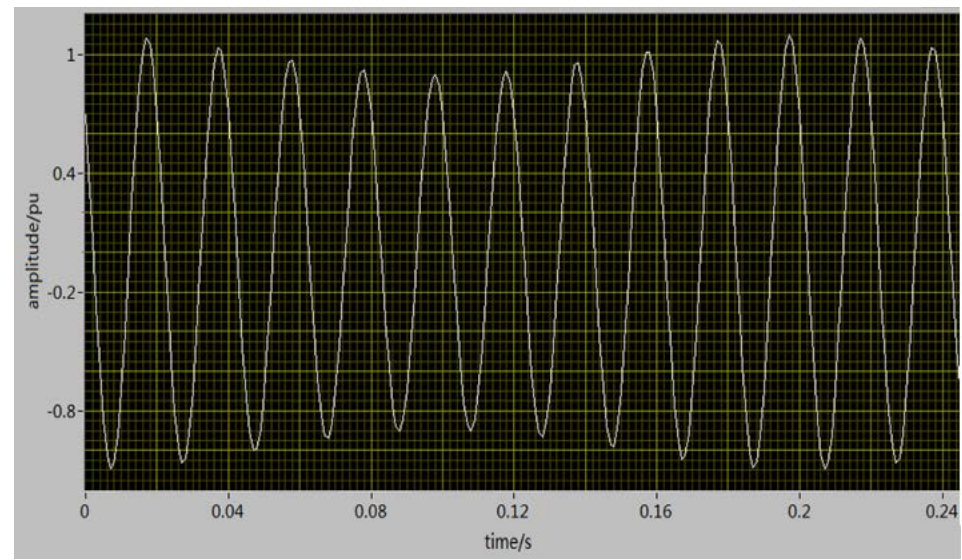

(b) Original power signal

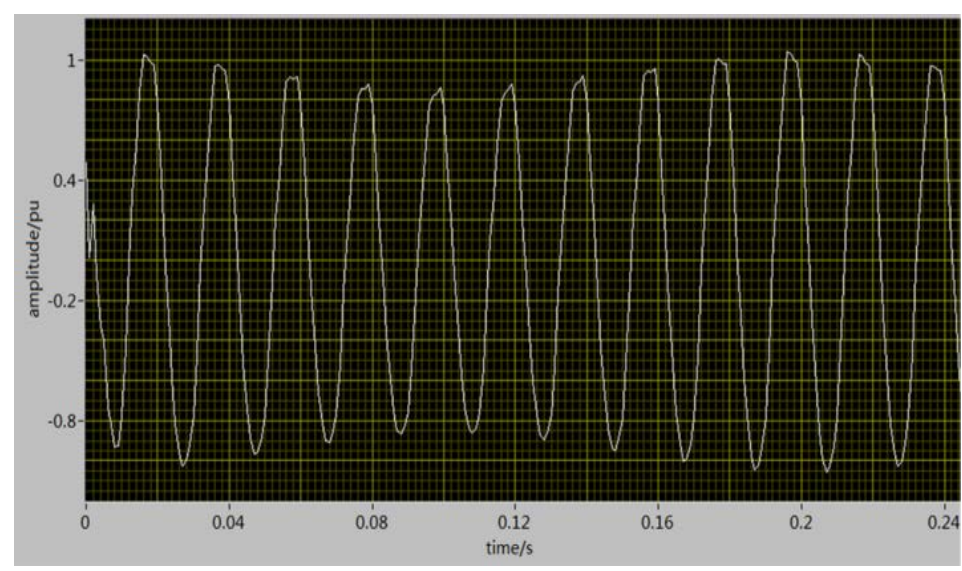

(c) Filter system output signal

Fig. 7. Filtering results of power signals with harmonics and noise 
The phasor measurement results of the CS algorithm, traditional DFT algorithm, and improved DFT algorithm for power signals with harmonics and noise are shown in Fig. 8. It can be seen that, during the initial stage of the phasor calculation, the traditional DFT algorithm has a large estimation error, which may be caused by slight fluctuations in the output of the filter system. However, even with the stability of the system output signal, a large error still exists in the phasor measurement, the TVE of traditional DFT algorithm exceeds $2 \%$. The improved DFT algorithm obviously reduces the estimation error of the amplitude and phase, and the TVE is only approximately 1\%. According to the phasor measurement results of the CS algorithm, the error values are smaller than those of the traditional and improved DFT algorithm, and tend to be stable. It can be seen that the proposed algorithm has good stability and a high measurement accuracy.

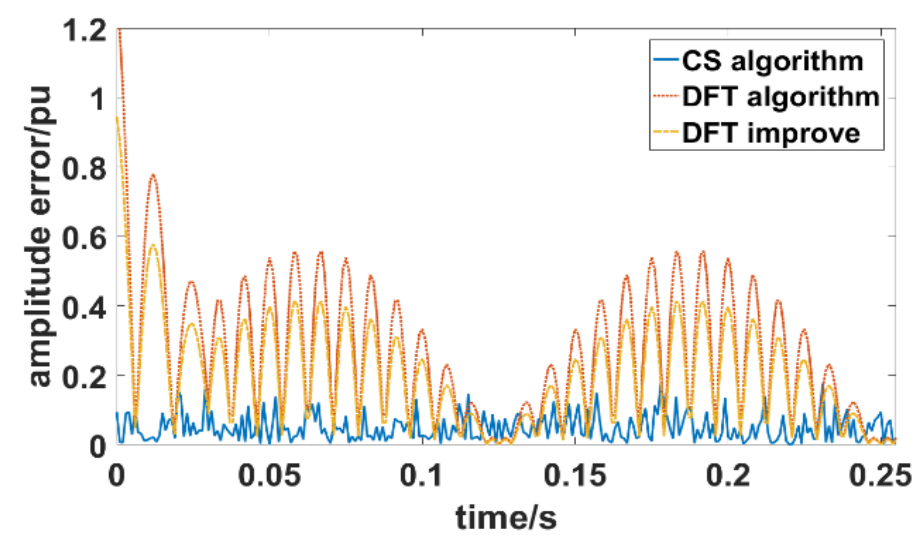

(a) Amplitude measurement error of power signals containing harmonics and noise

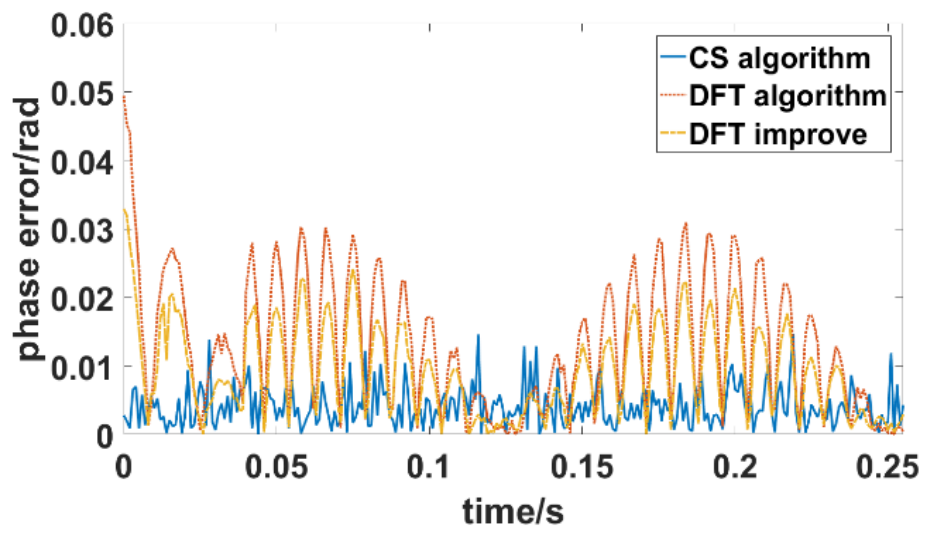

(b) Phase measurement error of power signals containing harmonics and noise 


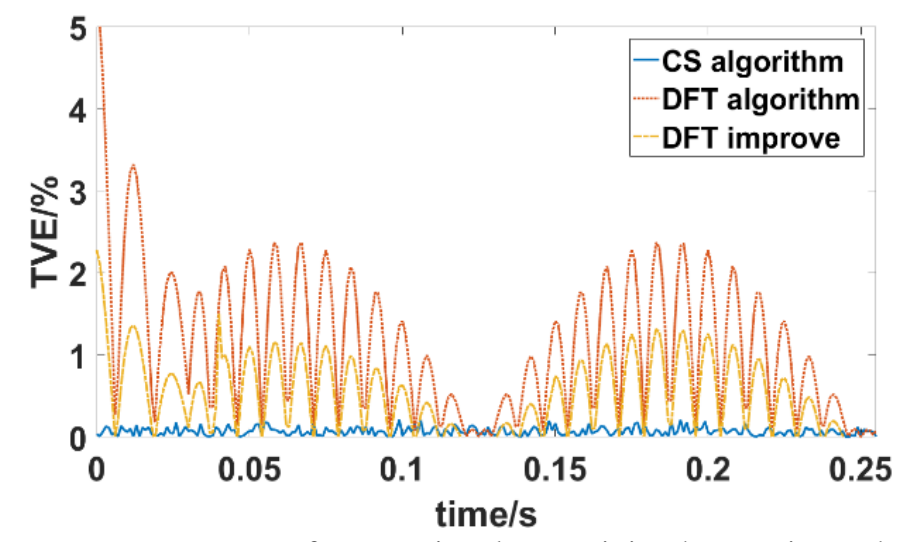

(c) TVE measurement of power signals containing harmonics and noise

Fig. 8. Measurement results for CS and DFT algorithms for harmonic signals with harmonics and noise

\subsection{Phasor measurement of amplitude step signals}

The voltage and current signals in the power system are affected by system disturbances and line faults in practical applications, resulting in a step change in the amplitude or phase of the power signal. To simulate this situation, the amplitude of the voltage signal at the rated power frequency $(50 \mathrm{~Hz}$ ) was stepped at $\mathrm{t}=0.1 \mathrm{~s}$, and the amplitude step test signal model was

$$
x(t)= \begin{cases}A_{m} \cdot \cos \left(2 \pi f_{0} t+\phi_{0}\right) & t<0.1 s \\ A_{t} \cdot \cos \left(2 \pi f_{0} t+\phi_{0}\right) & t \geqslant 0.1 s\end{cases}
$$

In the formula, $\phi_{0}=0.25 \pi$ is the initial phase, and $A_{m}=1$ represents the rated amplitude; in addition, $A_{t}$ is the magnitude of the amplitude after the mutation. According to the IEEE standard, in the amplitude step test, the step of the signal amplitude must be $10 \%$ of the rated amplitude, and therefore $A_{t}=1.1$. The amplitude step signal waveform is shown in Fig. 9.

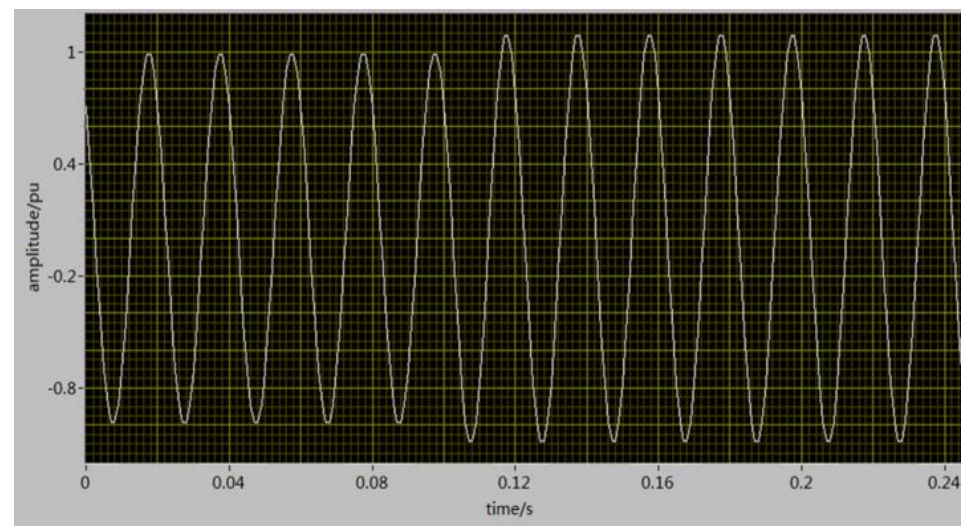

Fig. 9. Amplitude step signal waveform 
Fig. 10 shows the measurement error results of the CS algorithm, traditional DFT algorithm, and improved DFT algorithm for the amplitude step signal. It can be seen from Fig. 10 that, compared with the CS algorithm and improved DFT algorithm, the traditional DFT algorithm produces a large measurement error at the time of the amplitude step. The amplitude error, phase error, and TVE value of the CS algorithm are smaller than the traditional DFT and improved DFT algorithm within the $0-0.255$ s period. In addition, the error values of the CS algorithm tend to be stable. Therefore, the CS algorithm has a superior measurement accuracy and stability.

In addition, in the step response test, the most important performance indicator is the response time. The response time refers to the period from the time when the measurement error is beyond the precision range to the time when the measurement error is less than the precision range. In this test, the response time of the CS algorithm was $8 \mathrm{~ms}$, which satisfies the requirement of a response time of less than $30 \mathrm{~ms}$, whereas the response time of the traditional DFT algorithm is $64 \mathrm{~ms}$ and the response time of the improved DFT algorithm is $41 \mathrm{~ms}$. It can be seen that the step response performance of the CS algorithm is the best.

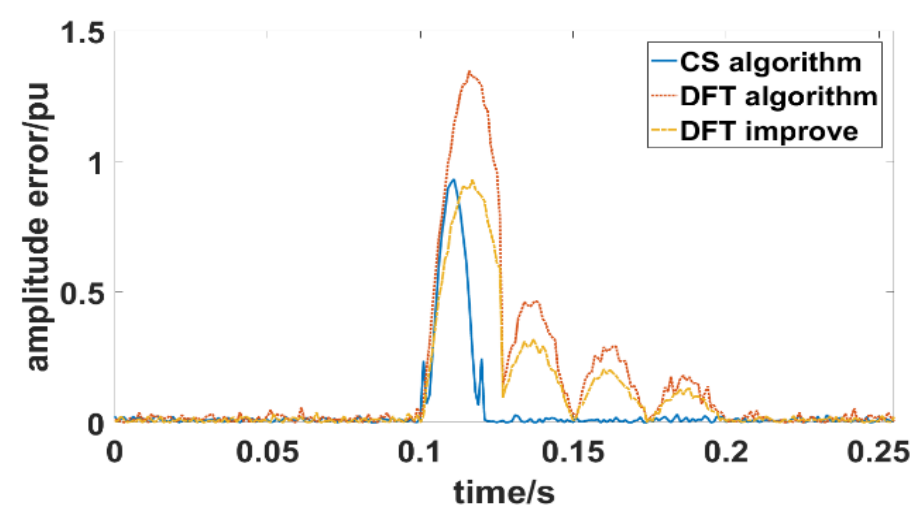

(a) Amplitude measurement error of the amplitude step signal

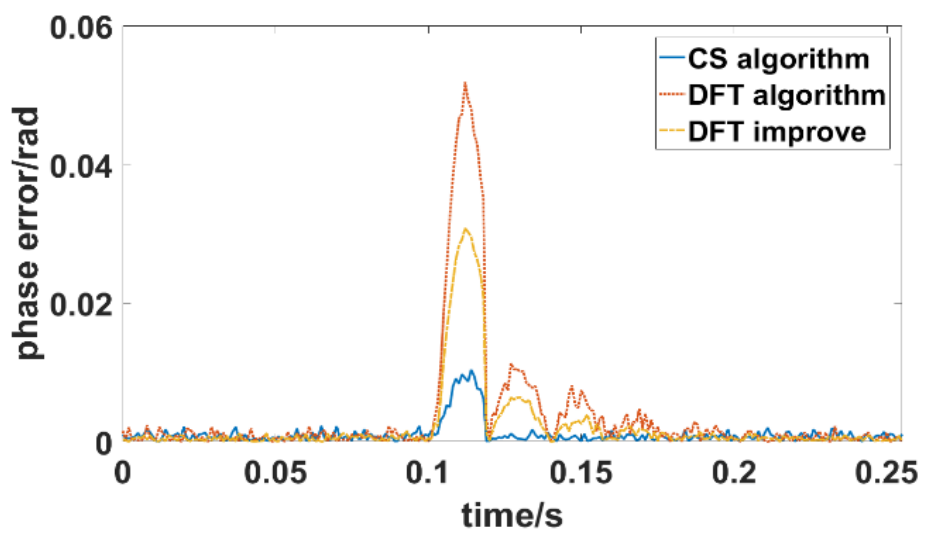

(b) Phase measurement error of the amplitude step signal 


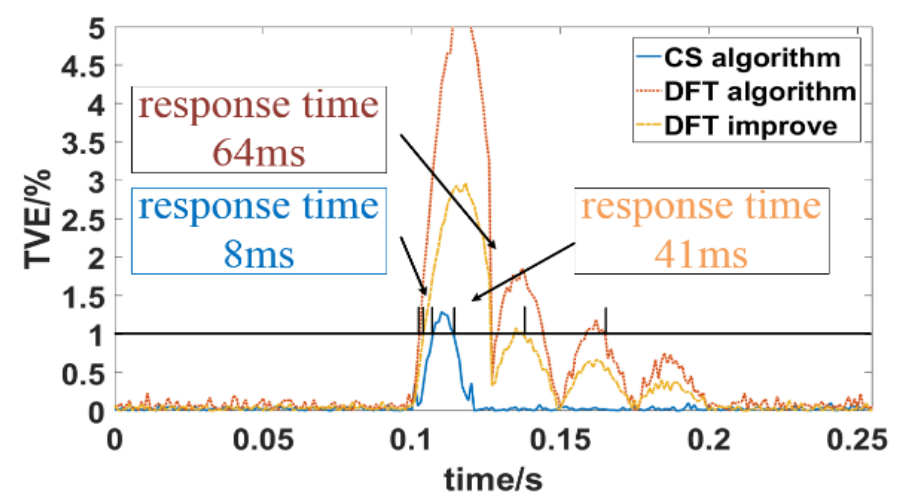

(c) TVE measurement of the amplitude step signal

Fig. 10. CS and DFT algorithm measurement results for amplitude step signals

\subsection{Phase measurement of phase step signals}

Similar to the amplitude step signal, the phase step signal is an abrupt change of phase. The test signal model is

$$
x(t)= \begin{cases}\cos \left(2 \pi f_{0} t+\phi_{1}\right) & t<0.1 s \\ \cos \left(2 \pi f_{0} t+\phi_{2}\right) & t \geqslant 0.1 s\end{cases}
$$

In the formula, $\phi_{1}=0.1 \pi$ and $\phi_{2}=0.2 \pi$. The test signal undergoes a phase change at $\mathrm{t}=0.1 \mathrm{~s}$ to achieve a phase step of the signal. The phase step signal waveform is shown in Fig. 11.

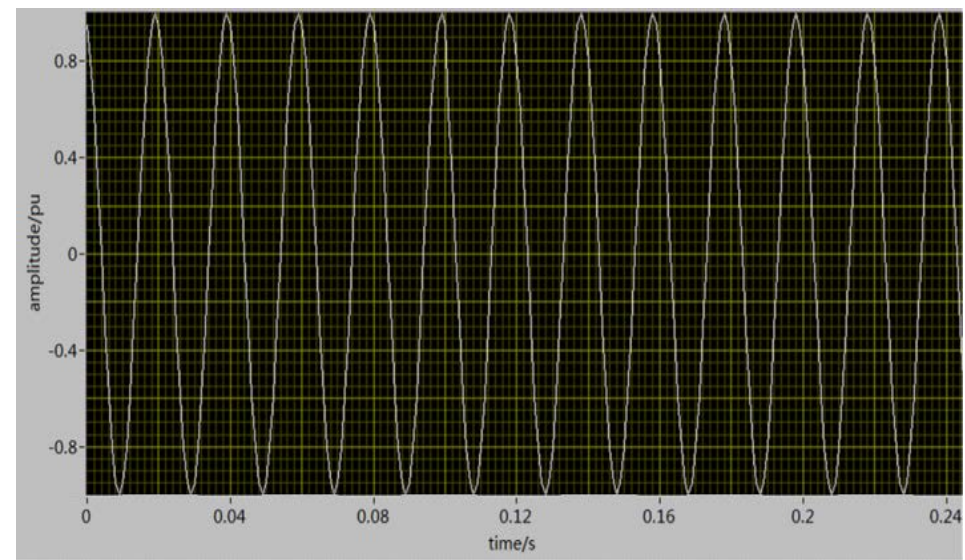

Fig. 11. Phase step signal waveform

For the phase step signal, Fig. 12 shows the measurement error results of the CS algorithm, traditional DFT algorithm, and improved DFT algorithm. It can be seen from Fig. 12 that, compared with the traditional and improved DFT algorithm, the measurement error of the CS algorithm is small within the entire signal mutation process, and the traditional DFT algorithm not only has a large measurement error at the phase step time, but also during the entire measurement period, creating a large fluctuation in the amplitude, phase error, and TVE value. Although the amplitude and phase measurement errors of the improved DFT 
algorithm are smaller than those of the traditional DFT algorithm, the accuracy is still lower than that of the CS algorithm, and therefore, it can be considered that the CS algorithm has a high measurement accuracy and good stability.

In addition, to further compare and analyze the performance of the three algorithms, the response times of the three algorithms were separately measured. As shown in Fig. 12, the measurement results of the proposed algorithm start to respond at $0.101 \mathrm{~s}$, the recovery convergence time is $0.119 \mathrm{~s}$, and the response time of the entire algorithm is $7 \mathrm{~ms}$, which satisfies the requirements of the phase step response time. The response time of the traditional DFT algorithm is $46 \mathrm{~ms}$, and the response time of the improved DFT algorithm is 29 ms, which are still longer than the response time of the CS algorithm, thus indicating that the step response performance of the proposed algorithm is better than both the traditional DFT algorithm and the improved DFT algorithm.

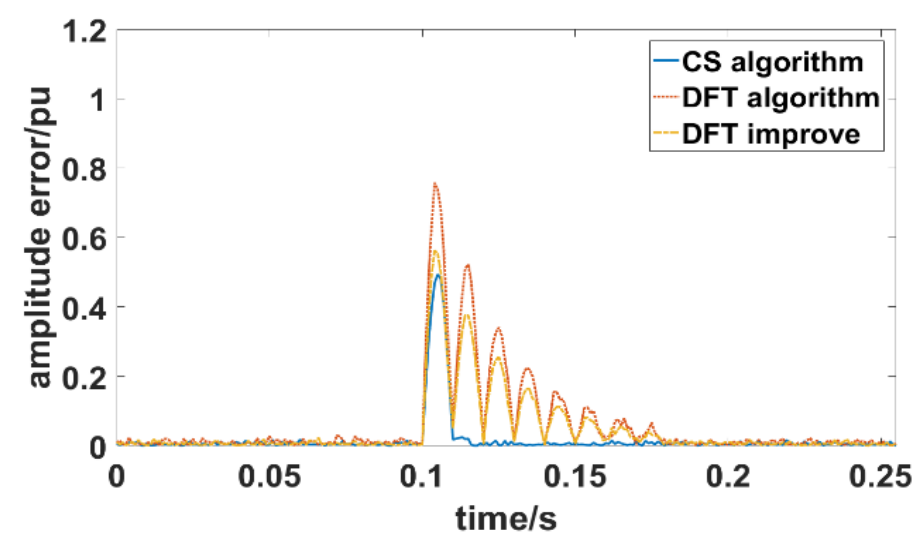

(a) Amplitude measurement error of the phase step signal

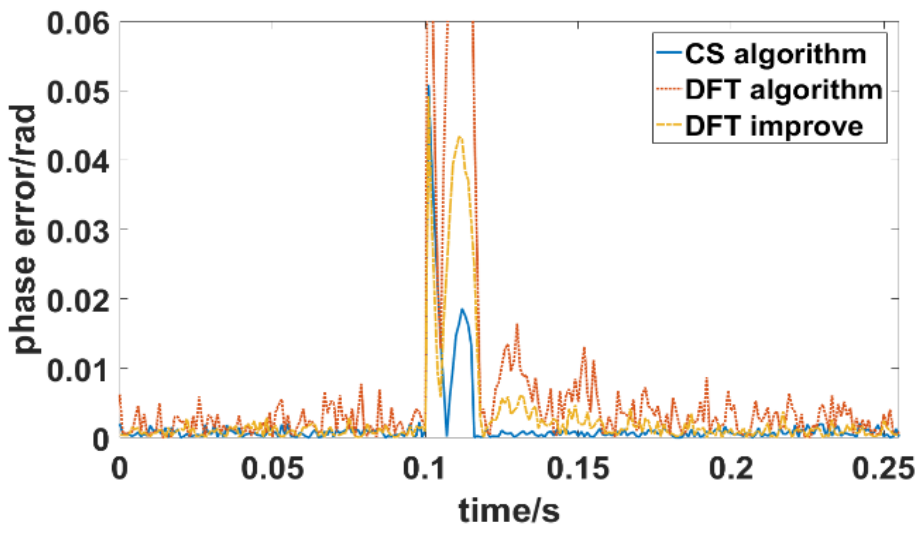

(b) Phase measurement error of the phase step signal 


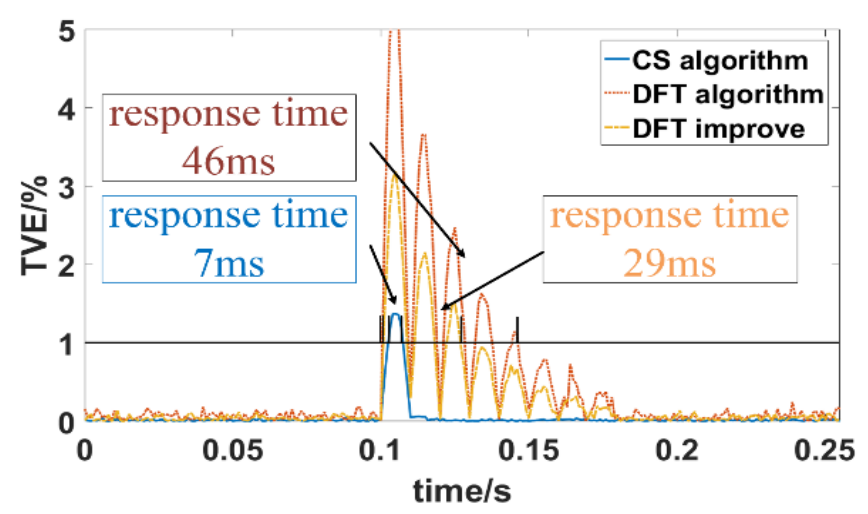

(c) TVE measurement of the phase step signal

Fig. 12. CS and DFT algorithm measurement results for phase step signals

\section{Future studies}

In a future study, we will continue to try to reduce the phasor measurement error of various signals through the compressed sensing algorithm, and continue to optimize the stability of the algorithm during the calculation process. The specific ideas are as follows:

1) Optimization of a sparse dictionary: To reduce the number of atoms needed during the sparse process and further improve the operation efficiency, we try to find the atoms that match the power signal better or optimize the parameters of the Chirplet atoms during the sparse process.

2) Optimization of signal filter: In this paper, the filter effectively filters out the harmonics and noise of the signal; however, the power system structure is complex, and the signal will be affected by a variety of complex perturbations, and thus we will optimize the filter to improve the ability of the signal to resist external interference.

\section{Conclusion}

In this paper, a dynamic synchronous phasor measurement algorithm based on compressive sensing was proposed. The algorithm was verified using the MATLAB simulation tool with reference to the IEEE standard. The following conclusions were obtained:

1) Compared with the traditional sparse dictionary of power signals, using the Chirplet dictionary to sparse the signal can greatly reduce the number of required atoms, reduce the computation time, and improve the computational efficiency.

2) In this study, the application of compressive sensing theory in the field of synchronous phasor measurement not only improves the phasor measurement accuracy of the power signals, but also effectively reduces the amount of sampled data, which is beneficial to the real-time transmission and processing of power signals.

3) By measuring the phasor of a low-frequency oscillation signal, a harmonic-containing power signal, an amplitude step signal, and a phase step signal, it was verified that the proposed synchronous phasor measurement algorithm based on compressive sensing has good dynamic conditions, being adaptive and consistent with the standards and requirements for a phasor measurement. 


\section{References}

[1] A. Ghasempour, "Advanced Metering Infrastructure in Smart Grid: Requirements, Challenges, Architectures, technologies, and Optimizations,” Smart Grids: Emerging Technologies, Challenges and Future Directions, Ed. J. Lou, Nova Science Publishers, 2017. Article (CrossRef Link)

[2] A. Ghasempour, "Internet of Things in Smart Grid: Architecture, Applications, Services, Key Technologies, and Challenges,” Inventions journal, vol. 4, no. 1, pp. 1-12, 2019. Article (CrossRef Link)

[3] A. Ghasempour, "Optimum number of aggregators based on power consumption, cost, and network lifetime in advanced metering infrastructure architecture for Smart Grid Internet of Things," in Proc. of 2016 13th IEEE Annual Consumer Communications \& Networking Conference (CCNC), Las Vegas, NV, pp. 295-296, 2016. Article (CrossRef Link)

[4] A. Ghasempour, "Optimized scalable decentralized hybrid advanced metering infrastructure for smart grid,” in Proc. of 2015 IEEE International Conference on Smart Grid Communications (SmartGridComm), Miami, FL, pp. 223-228, 2015. Article (CrossRef Link)

[5] A. Ghasempour, "Optimum Packet Service and Arrival Rates in Advanced Metering Infrastructure Architecture of Smart Grid," in Proc. of 2016 IEEE Green Technologies Conference (GreenTech), Kansas City, MO, pp. 1-5, 2016. Article (CrossRef Link)

[6] A. Ghasempour, "Optimized advanced metering infrastructure architecture of smart grid based on total cost, energy, and delay,” in Proc. of 2016 IEEE Power \& Energy Society Innovative Smart Grid Technologies Conference (ISGT), Minneapolis, MN, pp. 1-6, 2016.

Article (CrossRef Link)

[7] A. Ghasempour and T. K. Moon, “Optimizing the Number of Collectors in Machine-to-Machine Advanced Metering Infrastructure Architecture for Internet of Things-Based Smart Grid,” in Proc. of 2016 IEEE Green Technologies Conference (GreenTech), Kansas City, MO, pp. 51-55, 2016. Article (CrossRef Link)

[8] A. Ghasempour and J. H. Gunther, "Finding the optimal number of aggregators in machine-to-machine advanced metering infrastructure architecture of smart grid based on cost, delay, and energy consumption," in Proc. of 2016 13th IEEE Annual Consumer Communications \& Networking Conference (CCNC), Las Vegas, NV, pp. 960-963, 2016. Article (CrossRef Link)

[9] Jinquan Zhao, Yujie Zhang, Pan Zhang, Xiaoming Jin, Chao Fu, "Development of a WAMS based test platform for power system real time transient stability detection and control," Protection and Control of Modern Power Systems(PCMP), vol. 1, no. 1, 2016.

Article (CrossRef Link)

[10] Balimidi Mallikarjuna, Pudi Shanmukesh, Dwivedi Anmol, Maddikara Jaya Bharata Reddy, Dusmanta Kumar Mohanta, "PMU based adaptive zone settings of distance relays for protection of multi-terminal transmission lines," Protection and Control of Modern Power Systems(PCMP), vol. 3, no. 1, 2018. Article (CrossRef Link)

[11] S. D. Picard, M. G. Adamiak and V. Madani, "Fault location using PMU measurements and wide-area infrastructure," in Proc. of 2015 68th Annual Conference for Protective Relay Engineers, College Station, TX, pp. 272-277, 2015. Article (CrossRef Link)

[12] Mrinal Nandi, “On commutativity of Discrete Fourier Transform,” Information Processing Letters, vol. 115, no. 10, pp. 779-785, 2015. Article (CrossRef Link)

[13] D. Fan and V. Centeno, "Phasor-Based Synchronized Frequency Measurement in Power Systems,” IEEE Transactions on Power Delivery, vol. 22, no. 4, pp. 2010-2016, Oct 2007. Article (CrossRef Link)

[14] C. t. Nguyen and K. Srinivasan, “A New Technique for Rapid Tracking of Frequency Deviations Based on Level Crossings,” IEEE Transactions on Power Apparatus and Systems, vol. PAS-103, no. 8, pp. 2230-2236, Aug. 1984. Article (CrossRef Link)

[15] Prabhash Nanda, C.K. Panigrahi, Abhijit Dasgupta, "Phasor Estimation and Modelling Techniques of PMU- A Review,” Energy Procedia, vol. 109, pp. 64-77, 2017.

Article (CrossRef Link) 
[16] I. Kamwa, S. R. Samantaray and G. Joos, "Compliance Analysis of PMU Algorithms and Devices for Wide-Area Stabilizing Control of Large Power Systems," IEEE Transactions on Power Systems, vol. 28, no. 2, pp. 1766-1778, May 2013. Article (CrossRef Link)

[17] J. Follum and B. Amidan, "A data quality filter for PMU measurements: Description, experience, and examples," in Proc. of 2016 IEEE Power and Energy Society General Meeting (PESGM), Boston, MA, pp. 1-5, 2016. Article (CrossRef Link)

[18] Q. Wang, X. Yan and K. Qin, "High-Precision, Permanently Stable, Modulated Hopping Discrete Fourier Transform,” IEEE Signal Processing Letters, vol. 22, no. 6, pp. 748-751, June 2015. Article (CrossRef Link)

[19] S. Maharjan, J. C. H. Peng and J. E. Martinez, "Improved off-nominal operation of phasor measurement units using discrete fourier transformation," in Proc. of 2015 IEEE Power and Energy Conference at Illinois (PECI), Champaign, IL, pp. 1-5, 2015. Article (CrossRef Link)

[20] Jin tao, Chen yiyang, duan xiaohua, tang xiaoyan, "Research on Synchronous Phasor Measurement Algorithm of Power System Based on Improved DFT," Transactions of China Electrotechnical Society, vol. 32, no. 17, pp. 1-10, 2017. Article (CrossRef Link)

[21] W. Li and Y. Jia, "Distributed Gaussian sum filter for discrete-time nonlinear systems with Gaussian mixture noise," in Proc. of 2016 35th Chinese Control Conference (CCC), Chengdu, pp. 1831-1836, 2016. Article (CrossRef Link)

[22] J. Liu, F. Ni, P. A. Pegoraro, F. Ponci, A. Monti and C. Muscas, "Fundamental and harmonic synchrophasors estimation using modified Taylor-Kalman filter," in Proc. of 2012 IEEE International Workshop on Applied Measurements for Power Systems (AMPS) Proceedings, Aachen, pp. 1-6, 2012. Article (CrossRef Link)

[23] "PC37.118/D7.0 - Approved IEEE Draft Standard for Synchrophasors for Power Systems," IEEE Std PC37.118/D7.0, 2005. Article (CrossRef Link)

[24] Peijie Wang, Pooi-Yuen Kam, "An automatic step-size adjustment algorithm for LMS adaptive filters, and an application to channel estimation," Physical Communication, vol. 5, no. 3, pp. 280-286, 2012. Article (CrossRef Link)

[25] B. S. Shaik, G. V. S. S. K. R. Naganjaneyulu and A. V. Narasimhadhan, "A novel approach for QRS delineation in ECG signal based on chirplet transform," in Proc. of 2015 IEEE International Conference on Electronics, Computing and Communication Technologies (CONECCT), Bangalore, pp. 1-5, 2015. Article (CrossRef Link)

[26] J. A. Tropp and A. C. Gilbert, "Signal Recovery From Random Measurements Via Orthogonal Matching Pursuit," IEEE Transactions on Information Theory, vol. 53, no. 12, pp. 4655-4666, Dec 2007. Article (CrossRef Link)

[27] "C37.118.1-2011 - IEEE Standard for Synchrophasor Measurements for Power Systems," IEEE Std C37.118.1-2011 (Revision of IEEE Std C37.118-2005), pp.1-61, 28 Dec 2011.

Article (CrossRef Link) 


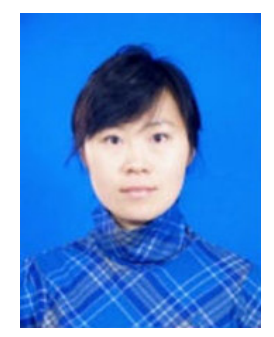

Huanan Yu received her B.Sc., M.Sc., and Ph.D. degrees from Jilin University in 2004, 2007, and 2012, respectively. She is currently an associate professor at Northeast Electric Power University. Her main research interests include compressed sensing and its applications in power systems, as well as power quality signal detection and analysis.

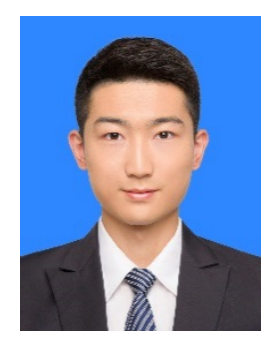

Yongxin Li is a graduate student in the M.Eng. program at Northeast Electric Power University. His main research interests are compressed sensing and its applications in power systems.

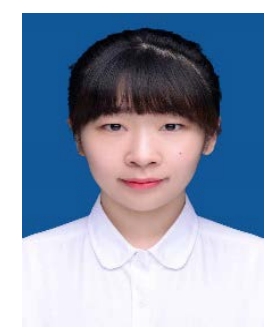

Yao Du received her master's degree from Northeast Electric Power University in 2018. She is currently working at the State Grid East Inner Mongolia Electric Power Supply Co., Ltd. Tongliao Power Supply Company, China. 